\title{
Glioma-targeting micelles for optical/magnetic resonance dual-mode imaging
}

\author{
This article was published in the following Dove Press journal: \\ International Journal of Nanomedicine \\ 5 March 2015 \\ Number of times this article has been viewed
}

\author{
Qing Zhoul,* \\ Ketao $\mathrm{Mu}^{2, *}$ \\ Lingyu Jiang' \\ Hui Xie ${ }^{3}$ \\ Wei Liu' \\ Zhengzheng $\mathrm{Li}^{\prime}$ \\ Hui Qi' \\ Shuyan Liang' \\ Huibi Xu' \\ Yanhong Zhu' \\ Wenzhen Zhu ${ }^{2}$ \\ Xiangliang Yang \\ 'National Engineering Research \\ Center for Nanomedicine, College \\ of Life Science and Technology, \\ ${ }^{2}$ Radiology Department, Tongji \\ Hospital, Tongji Medical College, \\ Huazhong University of Science \\ and Technology, ${ }^{3}$ Department of \\ Information Processing, China Patent \\ Information Center, Wuhan, People's \\ Republic of China \\ *These authors contributed equally \\ to this work
}

\section{Correspondence: Xiangliang Yang National Engineering Research Center for Nanomedicine, College of Life Science and Technology, Huazhong University of Science and Technology, Wuhan 430074, People's Republic of China \\ Tel +86 I38 07172067 \\ Fax +862787792234 \\ Email yangx|@hust.edu.cn}

Wenzhen Zhu

Radiology Department of Tongji Hospital,

Tongji Medical College, Huazhong

University of Science and Technology,

Wuhan 430030, People's Republic of

China

Tel +86 I38 860। 86I2

Fax +862783663258

Email zhuwenzhen@hotmail.com

\begin{abstract}
Surgical resection is the primary mode for glioma treatment, while gross total resection is difficult to achieve, due to the invasiveness of the gliomas. Meanwhile, the tumorresection region is closely related to survival rate and life quality. Therefore, we developed optical/magnetic resonance imaging (MRI) bifunctional targeted micelles for glioma so as to delineate the glioma location before and during operation. The micelles were constructed through encapsulation of hydrophobic superparamagnetic iron oxide nanoparticles (SPIONs) with polyethylene glycol-block-polycaprolactone (PEG- $b$-PCL) by using a solvent-evaporation method, and modified with a near-infrared fluorescent probe, Cy5.5, in addition to the gliomatargeting ligand lactoferrin (Lf). Being encapsulated by PEG- $b$-PCL, the hydrophobic SPIONs dispersed well in phosphate-buffered saline over 4 weeks, and the relaxivity $\left(r_{2}\right)$ of micelles was $215.4 \mathrm{mM}^{-1} \cdot \mathrm{s}^{-1}$, with sustained satisfactory fluorescent imaging ability, which might have been due to the interval formed by PEG- $b$-PCL for avoiding the fluorescence quenching caused by SPIONs. The in vivo results indicated that the nanoparticles with Lf accumulated efficiently in glioma cells and prolonged the duration of hypointensity at the tumor site over 48 hours in the MR image compared to the nontarget group. Corresponding with the MRI results, the margin of the glioma was clearly demarcated in the fluorescence image, wherein the average fluorescence intensity of the tumor was about fourfold higher than that of normal brain tissue. Furthermore, 3-(4,5-dimethyl-2-thiazolyl)-2,5-diphenyl-2-H-tetrazolium bromide assay results showed that the micelles were biocompatible at Fe concentrations of $0-100 \mu \mathrm{g} / \mathrm{mL}$. In general, these optical/ MRI bifunctional micelles can specifically target the glioma and provide guidance for surgical resection of the glioma before and during operation.
\end{abstract}

Keywords: MRI, fluorescence image, micelles, lactoferrin, glioma

\section{Introduction}

Gliomas are the most frequently occurring type of primary brain tumors in Europe and the US. ${ }^{1}$ Due to their high malignancy, the survival rate of patients is only $5 \%$ after 5 years. $^{2}$ The current standard therapy for glioma is maximal safe resection followed by chemoradiotherapy or photodynamic therapy. ${ }^{1,2}$ However, it is quite difficult to achieve a gross total resection of the brain tumor without damage to normal brain tissue, owing to the infiltration and invasiveness of the glioma, which seriously affects the survival rate and life quality. ${ }^{2,3}$ Therefore, it is of great importance to develop a new method for defining the margin of gliomas to provide guidance for preoperation planning and intraoperation navigation.

Preoperative imaging on the tumor with magnetic resonance imaging (MRI) is currently widely applied for brain-glioma diagnosis, and is commonly used for operation planning. ${ }^{4,5}$ Gadolinium $\left(\mathrm{Gd}^{3+}\right)$-based contrast agents, such as $\mathrm{Gd}^{3+}$-diethylenetriaminepentaacetic acid (Magnevist), are always used for enhancing the signal at the tumor site. ${ }^{6}$ However, $\mathrm{Gd}^{3+}$-based contrast agents are rapidly excreted within 24 hours, due

submit your manuscript | www.dovepress.com 
to the glomerular filtration effect, and then provide a short duration of the enhanced signal. ${ }^{7}$ Compared to the traditional $\mathrm{Gd}^{3+}$-based contrast agents, another commonly-used MRI contrast agent - superparamagnetic iron oxide (SPIO) - has characteristics of high sensitivity, lower toxicity, and excellent biocompatibility. Nevertheless, directly prepared SPIO is generally an oil-soluble material so as not to be adopted by organisms. ${ }^{89}$ Currently, a number of methods, such as encapsulation of the SPIO with chitosan or polymers, are being developed to convert oil-soluble SPIO into watersoluble SPIO, wherein the amount of the SPIO cleared by the reticuloendothelial system is reduced with modifications, leading to prolonged blood circulation in vivo, and especially SPIO encapsulated by amphiphilic polymer shows good stability, which is gaining a lot of attention. ${ }^{10-13}$

For improved resection rate and surgery safety, intraoperative imaging is equally important as preoperative imaging. In Kubben at al's study, intraoperative MRI-guided glioblastoma multiforme-resection results showed that compared with conventional neuronavigation-guided surgery, intraoperative MRI-guided surgery provided better results for tumor resection, life-quality improvement, and prolonged postoperative survival time, while intraoperative MRI required repeated imaging during the surgery to determine the tumor region, which, unfortunately, might increase the possibility of infection for patients. ${ }^{14}$ Compared to intraoperative MRI, fluorescence imaging has advantages of high sensitivity, convenience, and low cost. ${ }^{15,16} \mathrm{In}$ Tsugu et al's study, comparison of intraoperative MRI and the 5-aminolevulinic acid (5-ALA)-guided fluorescence imaging showed that for 5-ALA-induced fluorescence-positive gliomas (including almost all the malignant gliomas), good resection effects were obtained only with 5-ALA fluorescence imagingguided resection. ${ }^{17}$ However, conventional 5-ALA fluorescence imaging-guided surgery had a plurality of side effects, such as skin photosensitivity, and protoporphyrin IX was not produced in all the gliomas, especially in low-grade gliomas, after oral administration of the 5-ALA. ${ }^{18,19}$ Small-molecule fluorescent probes have been applied widely in biomedical fields, due to their good optical properties and excellent biocompatibility, wherein the near-infrared fluorescent (NIRF) dye is generally adopted for optical imaging in vivo so as to minimize autofluorescence of the tissue and acquire deep penetration. Among various NIRF dyes, Cy5.5 is widely used in the biological field, due to its high molar absorbancy index, high fluorescence quantum yield, and no photosensitivity. ${ }^{20,21}$

For these reasons, it is imperative to develop a low-toxicity contrast agent for preoperation planning and intraoperation navigation of gliomas. Based on our previous research, ${ }^{22}$ we developed a contrast agent - Cy5.5-lactoferrin (Lf)-SPIO micelles - targeting gliomas and performing optical/MR imaging, wherein SPIO was encapsulated with an amphiphilic polymer polyethylene glycol-block-polycaprolactone (PEG$b$-PCL) to form magnetic micelles, Cy5.5 was selected as an NIRF probe, Lf and Cy5.5 were linked to the amino terminal of the amphiphilic polymer PEG- $b$-PCL, and Cy5.5 and SPIO were separated with the amphiphilic polymer PEG- $b$-PCL to avoid fluorescence quenching caused by SPIO. The Cy5.5-Lf-SPIO micelles had good dispersity and high colloidal stability in phosphate-buffered saline (PBS). In vitro study results showed that the Cy5.5-Lf-SPIO micelles were specifically taken up by $\mathrm{C} 6$ glioma cells. In vivo study results indicated that the Cy5.5-Lf-SPIO micelles targeted the C6 rat glioma, had good optical/MR-imaging function, and provided clear delineation of the boundary of the glioma.

\section{Materials and methods Materials}

Cy5.5- $N$-hydroxysuccinimide (NHS) was purchased from GE Healthcare (Piscataway, USA). Lf from bovine colostrum was purchased from Sigma-Aldrich (St Louis, USA). Methoxy-PEG $\mathrm{PE00}_{50}-\mathrm{PCL}_{15000}$ was purchased from Daigang Biomaterial (Shandong, People's Republic of China). $\mathrm{NH}_{2}-$ $\mathrm{PEG}_{5800}-b-\mathrm{PCL}_{19000}$ was purchased from Polymer Source (Montreal, Canada). $\mathrm{N}$-succinimidyl iodoacetate (SIA) and Traut's reagent were purchased from Thermo Fisher Scientific (Waltham, USA).

\section{Synthesis of bifunctional micelles}

Oleylamine (OAm)-coated $\mathrm{Fe}_{3} \mathrm{O}_{4}$ nanoparticles were produced through the thermal decomposition method. ${ }^{9}$ The SPIO micelles were prepared by adding a copolymer and SPIO mixture into pure water in a dropwise manner under a vigorous ultrasonic condition. Then, the SPIO micelles were mixed with Cy5.5 NHS ester. Lf reacted with Traut's agent to be subjected for primary functionalization with sulfhydryl groups. The Cy5.5SPIO micelles were conjugated with SIA. The functionalized Lf was then conjugated to the Cy5.5-SPIO micelles via iodoacetate. The complete process is shown in Figure 1.

\section{Synthesis of OAm magnetic nanoparticles}

Monodispersed OAm magnetic nanoparticles were synthesized according to the thermal decomposition method. ${ }^{9}$ $\mathrm{Fe}(\mathrm{acac})_{3}(2 \mathrm{mmol})$ was dissolved in a mixture of $10 \mathrm{~mL}$ of benzyl ether and $10 \mathrm{~mL}$ of OAm. The solution was stirred magnetically in the presence of $\mathrm{N}_{2}$ with a constant flow, then heated gradually to $110^{\circ} \mathrm{C}\left(5^{\circ} \mathrm{C} /\right.$ minute $)$ and dehydrated for 

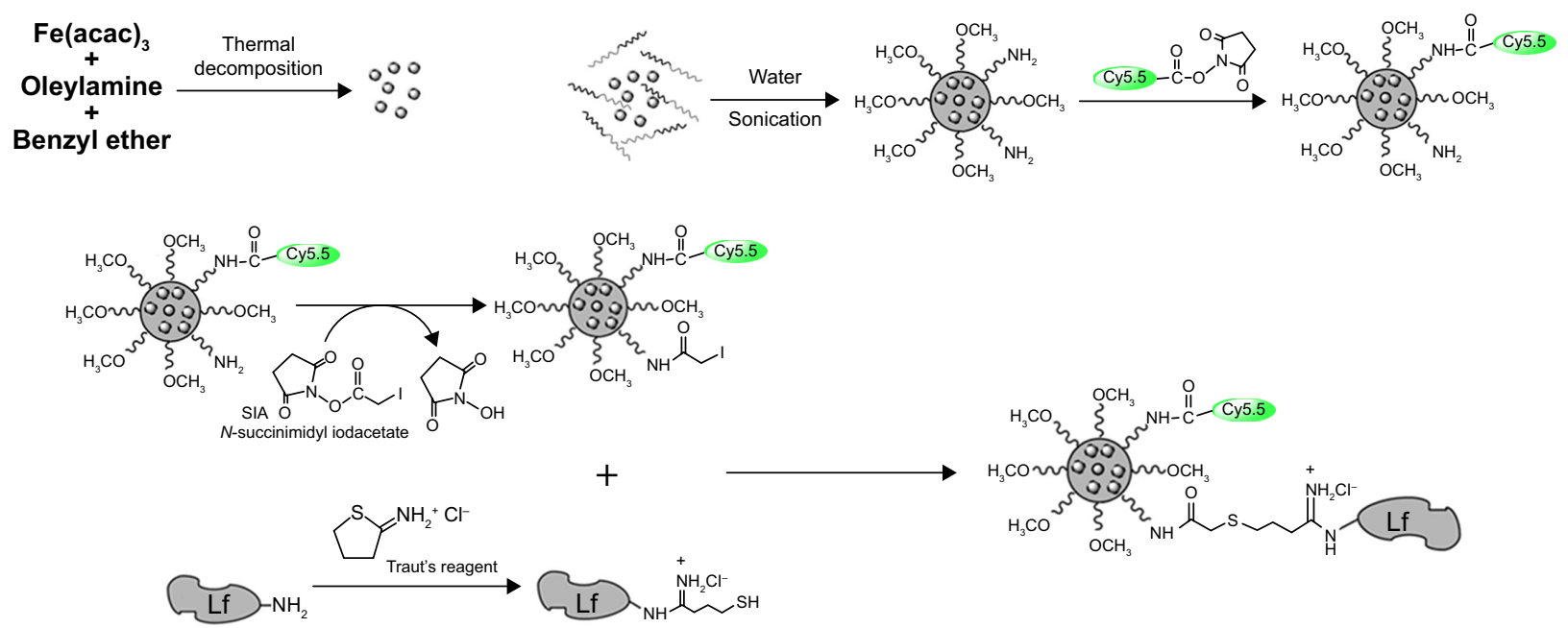

Figure I Steps for preparation of the Cy5.5-Lf-SPIO micelles.

Abbreviations: Lf, lactoferrin; SPIO, superparamagnetic iron oxide; SIA, succinimidyl iodoacetate.

1 hour. After that, the mixture was heated to achieve a reflux temperature $\left(\sim 300^{\circ} \mathrm{C}\right)$ at a heating rate of $10^{\circ} \mathrm{C} /$ minute and aged at this temperature for 1 hour. After the mixture cooled down to room temperature, $50 \mathrm{~mL}$ of absolute ethanol was added into the black mixture. The precipitation was collected through centrifuge (7,000 rpm, 10 minutes) and was washed with ethanol three times. Finally, OAm magnetic nanoparticles were redispersed in tetrahydrofuran (THF).

\section{Formation of SPIO-loaded polymeric micelles}

PEG- $b$-PCL copolymer ( $3 \mathrm{mg}$, containing $0.5 \mathrm{mg} \mathrm{NH}_{2}-$ $\mathrm{PEG}_{5800}-b-\mathrm{PCL}_{19000}$ and $2.5 \mathrm{mg}$ methoxy-PEG $\left.\mathrm{P}_{500}-b-\mathrm{PCL}_{15000}\right)$ and $9 \mathrm{mg}$ SPIO nanoparticles $(\sim 8 \mathrm{~nm})$ were first dissolved in $2.5 \mathrm{~mL}$ of THF in a glass vial. Then, the mixture was added to pure water $(25 \mathrm{~mL})$ in a dropwise manner under a vigorous ultrasonic condition by using a JY92-II N (Scientz) at a power of $200 \mathrm{~W}(2 \mathrm{~s} / 2 \mathrm{~s})$. Then, the THF in the solution was evaporated by vacuum distillation. The SPIO-loaded micelle samples were stored at $4^{\circ} \mathrm{C}$, and all related measurements were taken within 2 weeks.

\section{Modification of SPIO-loaded polymeric micelles}

Monoreactive Cy5.5 NHS ester (1 mg) was dissolved in $50 \mu \mathrm{L}$ of dimethyl sulfoxide (DMSO). Five microliters of the obtained solution was added to $2 \mathrm{~mL}$ of SPIO-loaded micelles ( $3 \mathrm{mg}$ of Fe/mL). After 2 hours of suspension reaction, $2 \mathrm{mg}$ SIA, which was dissolved in $0.1 \mathrm{~mL}$ of DMSO, was added to this. The solution was protected from light. Then, it was put on a shaker for 2 hours at room temperature. After that, a magnet was used to separate the reaction solution, and the supernatant was abandoned. Simultaneously, a sulfhydryl group was attached to the Lf via primary amine modification through treatment with Traut's reagent. Moreover, $2 \mathrm{mg}$ Traut's reagent was dissolved in $1 \mathrm{~mL}$ borate buffer. The borate buffer was made of disodium tetraborate. The concentration was $0.15 \mathrm{M}$ and the $\mathrm{pH}$ was 8.5 . Then, $0.5 \mathrm{~mL}$ of Traut's reagent stock solution was mixed with $5 \mathrm{mg} \mathrm{Lf}(10 \mathrm{mg} / \mathrm{mL}$ Lf dissolved in a borate buffer), and the obtained solution was further reacted for 1 hour at room temperature. After the thiolation reaction, micelles were separated from the reaction solution using a magnet, and the supernatant was discarded.

\section{Characterizations of Cy5.5-Lf-SPIO micelles}

The size and morphology of the Cy5.5-Lf-SPIO micelles were assessed by transmission electron microscopy (TEM; JEM-2010; JEOL, Tokyo, Japan) at $200 \mathrm{kV}$. Dynamic light scattering (Zetasizer Nano ZS90; Malvern Instruments, Malvern, UK) was used to measure the hydrodynamic diameter of the nanoparticles with an angle of $90^{\circ}$.

Fourier-transform IR spectroscopy (Vertex 70; Bruker, Ettlingen, Germany) was used to achieve qualitative analysis of the Lf conjugation. The bicinchoninic acid assay was used to achieve quantitative analysis of the Lf conjugation.

The relaxivity of the materials was measured with the method mentioned in our previous work. ${ }^{23}$ The micelles were diluted in distilled water with iron concentrations of $0-2.5 \mu \mathrm{g} / \mathrm{mL}$. Samples were transferred to a 96-well plate, and $T_{2}$ relaxation times of the suspensions were determined with a $3.0 \mathrm{~T}$ 
whole-body MR scanner (Signa HDxt 3.0 T; GE) in combination with an eight-channel head coil. The parameters were optimized as follows: field of view was $120 \mathrm{~mm}$, base resolution was $192 \times 160$, slice thickness was $1.5 \mathrm{~mm}$, interval thickness was $0.5 \mathrm{~mm}$, multiple echo times were 20, 40, 60, $80,100,120$, and $140 \mathrm{~ms}$, and repetition time was $2,000 \mathrm{~ms}$. $T_{2}$ relaxation rates were plotted against iron concentrations in the particle dilutions set. Relaxivity was determined through a linear fitting model.

Magnetic properties were measured with a vibrating sample magnetometer (model 7404; Lake Shore Cryotronics, Westerville, USA) under a maximum field of $15 \mathrm{kOe}$ at room temperature. To evaluate the fluorescence property of the materials, a fluorescence spectrophotometer (FL4500; Hitachi, Tokyo, Japan) was used. The excitation wavelength was $640 \mathrm{~nm}$.

An IVIS Lumina XR system (Caliper Life Sciences, Hopkinton, USA) was used to measure the fluorescence property of the materials. The applied method was the same as in our previous work. ${ }^{23}$ Briefly, the micelles were diluted at different Fe concentrations ranging from 0 to $300 \mu \mathrm{g} / \mathrm{mL}$. The excitation wavelength was $640 \mathrm{~nm}$, and the emission wavelength was set at Cy5.5's wavelength.

\section{In vitro studies} Incubation

C6 glioma cells (C6) were plated in a six-well plate. Cy5.5Lf-SPIO-loaded micelles and Cy5.5-SPIO-loaded micelles (dissolved in Dulbecco's Modified Eagle's Medium) at different iron concentrations ranging from 0 to $25 \mu \mathrm{g} / \mathrm{mL}$ were added to the plate. After incubation for 1.5 hours, the cells were washed with PBS (0.1 M, pH 7.4) three times.

\section{In vitro MRI}

After being washed, the cells were collected and placed in a 96-well plate. The plate was scanned by the $3.0 \mathrm{~T}$ wholebody MR scanner.

\section{Confocal microscopy}

After being washed, the cells were stained with 4',6diamidino-2-phenylindole (DAPI; $5 \mu \mathrm{g} / \mathrm{mL}$ ) for 20 minutes. Then, the fluorescence images of the cells were collected on a laser scanning confocal microscope (TCS-SP5; Leica, Wetzlar, Germany) by using a $40 \times$ objective. For Cy5.5, the excitation wavelength was $633 \mathrm{~nm}$, and the emissionwavelength range was $650-750 \mathrm{~nm}$. For DAPI, the excitation wavelength was $405 \mathrm{~nm}$, and the emission-wavelength range was $420-480 \mathrm{~nm}$.

\section{In vitro fluorescence imaging}

After being washed, the cells were collected and placed in a 96-well plate. The IVIS Lumina XR system was used to collected fluorescent images. The excitation wavelength was $640 \mathrm{~nm}$, and the emission wavelength was set at Cy5.5's wavelength.

\section{In vivo studies}

\section{Animal-model preparation}

The animal model was prepared via the method mentioned in our previous work. ${ }^{22}$ Rats were anesthetized with $10 \%$ chloral hydrate (intraperitoneally, $0.4 \mathrm{~mL} / 100 \mathrm{~g}$ body weight) and placed in a stereotactic frame. A burr hole was drilled into the skull (1.0 mm posteriorly and $3.0 \mathrm{~mm}$ laterally to the bregma). A $50 \mu \mathrm{L}$ microinjector was used to inject $10 \mu \mathrm{L}$ of $1 \times 10^{6} \mathrm{C} 6$ cells suspended in the serum-free Dulbecco's Modified Eagle's Medium. The injection was performed slowly over 10 minutes. Once the injection was completed, the needle stayed in the brain for another 10 minutes. The needle was then withdrawn from the brain slowly. The skin was closed with nonmagnetic sutures. MRI and fluorescence imaging were performed after tumors grew to $4 \times 4 \mathrm{~mm}^{2}$ (about 11 days). The procedure followed the previously published report. ${ }^{22}$

\section{In vivo MRI}

One milliliter of PBS (0.1 M, pH 7.4) containing Cy5.5-LfSPIO micelles or Cy5.5-SPIO micelles (12 mg Fe/kg body weight) ( $n=6)$ was administered via the tail vein. A 3.0 T MR system (Signa HDxt) was used to collect MR images at 24 and 48 hours postinjection. The parameters were set as mentioned in our previous work: ${ }^{23}$ field of view was $60 \mathrm{~mm}$, base resolution was $192 \times 160$, slice thickness was $1.5 \mathrm{~mm}$, interval thickness was $0.5 \mathrm{~mm}$, multiple echo time was $50 \mathrm{~ms}$, and repetition time was 3,000 ms. The signal intensity of the tumor and the muscle were measured. Relative signal enhancement (RSE) was calculated according to the following formula: ${ }^{24}$

$\operatorname{RSE}(\%)=\left(1-\left[\mathrm{SI}_{\text {post,tumor }} / \mathrm{SI}_{\text {post,muscle }}\right] /\left[\mathrm{SI}_{\text {pre,tumor }} / \mathrm{SI}_{\text {pre,muscle }}\right]\right) \times 100$

$\mathrm{SI}_{\text {pre }}$ was measured before injection of the nanoparticles, and $\mathrm{SI}_{\text {post }}$ was measured at each follow-up time after injection (24 and 48 hours).

\section{Ex vivo fluorescence imaging}

For ex vivo study, the rats were killed. The IVIS Lumina XR system was used to collect the fluorescence images of the rats' brain tissues. The excitation wavelength was $640 \mathrm{~nm}$, and the emission wavelength was set at Cy5.5's wavelength. 


\section{Histology study}

After brain fixation and dehydration, each rat's brain was sectioned into slices with a thickness of $10 \mu \mathrm{m}$. The slices of each brain were divided into three groups. Group 1 was stained with hematoxylin and eosin (H\&E), group 2 was stained with DAPI, and group 3 was stained with Prussian blue and nuclear fast red solution. Confocal images of the slices stained with DAPI were collected through the laser scanning confocal microscope with a $10 \times$ objective. For Cy5.5, the excitation wavelength was $633 \mathrm{~nm}$, and the emission wavelength range was from $650 \mathrm{~nm}$ to $750 \mathrm{~nm}$. For DAPI, the excitation wavelength was $405 \mathrm{~nm}$, and the emission wavelength range was from $420 \mathrm{~nm}$ to $480 \mathrm{~nm}$.

\section{In vitro cytotoxicity}

The mouse embryo fibroblast cell line NIH-3T3 was plated in a 96-well plate at $5 \times 10^{3}$ cells/well. The cells were treated with the medium containing Cy5.5-Lf-SPIO micelles at different Fe concentrations ranging from 0 to $100 \mu \mathrm{g} / \mathrm{mL} \mathrm{Fe}$. The control well was a culture medium without the nanoparticles. After 24, 48, and 72 hours of incubation, the in vitro cytotoxicity was evaluated by 3-(4,5-dimethyl-2-thiazolyl)-2, 5-diphenyl-2-H-tetrazolium (MTT) assay.

\section{Statistical analysis}

The data are expressed as means \pm standard error of the mean. A difference of $P<0.05$ was considered statistically significant, and $P<0.01$ was considered highly statistically significant. Statistical analysis was analyzed with Student's $t$-test using statistical software (SPSS 17.0; SPSS, Chicago, USA).

\section{Results}

\section{Characterization of Cy5.5-Lf-SPIO micelles}

The TEM images (Figure 2B) indicated that the SPIO, just as Figure 2A shows, was encapsulated into the micelles and formed a cluster in the hydrophobic core of the micelles. As shown in the TEM image, the mean diameter of the particles was measured as $92.1 \pm 4.8 \mathrm{~nm}$. Meanwhile, the mean hydrodynamic size and zeta potential of the Cy5.5-Lf-SPIO micelles were $390.4 \pm 10.4 \mathrm{~nm}$ (polydispersity index $=0.083$ ) (Figure 2C) and $-2.53 \mathrm{mV}$, respectively, by dynamic light scattering. The difference between the hydrodynamic size and the size shown in the TEM images may have been due to the copolymer and Lf increasing the hydrodynamic diameter of the nanoparticles. As shown in the Fouriertransform IR spectra (Figure S1), in the pure Lf group and
Cy5.5-Lf-SPIO micelle group, the bands around 3,400 $\mathrm{cm}^{-1}$ were for acylamino-stretching vibration, which indicated that the Lf existed. In the Cy5.5-Lf-SPIO micelle group, the bands around $700 \mathrm{~cm}^{-1}$ appeared to be due to $\mathrm{S}-\mathrm{C}$ bond formation, which indicated that Lf successfully conjugated to micelles. The bicinchoninic acid assay was used to quantify the $\mathrm{Lf}$ conjugation. There were $3.3 \times 10^{-3} \mathrm{mg}$ Lf per milligram Fe. Moreover, long-term dynamic light-scattering measurements showed that there was no significant particle size change or flocculation of the Cy5.5-Lf-SPIO micelles dissolved in PBS for 4 weeks, demonstrating that the micelles were highly stable in PBS (Figure 2D).

To evaluate the magnetic properties of the Cy5.5-LfSPIO micelles, the nanoparticles were examined by a vibrating sample magnetometer. Figure $3 \mathrm{~A}$ shows that both Cy5.5-Lf-SPIO micelles and SPIO were superparamagnetic at $300 \mathrm{~K}$. The saturation magnetizations of Cy5.5-Lf-SPIO micelles and SPIO were $67.4 \mathrm{emu} / \mathrm{g} F e$ and $76.2 \mathrm{emu} / \mathrm{g} \mathrm{Fe}$ respectively, which indicated that the copolymer and the modification of the nanoparticles affected the magnetic properties of the materials slightly. To acquire the $T_{2}$ relaxation $\left(r_{2}\right)$ of the Cy5.5-Lf-SPIO micelles, the relaxation times of Cy5.5-Lf-SPIO micelles with various iron concentrations were measured by 3.0 T MRI. The results showed a good linear correlation between $T_{2}$ relaxation $\left(r_{2}\right)$ and iron concentration, with $r_{2}=215.4 \mathrm{mM}^{-1} \cdot \mathrm{s}^{-1}$ (Figure 3B and C), which suggested that the nanoparticles could be utilized as a negative MRI contrast agent in $T_{2}$-weighted imaging.

Among various fluorescent dyes, Cy5.5 has been widely used in biomedical fields, due to its high molar absorption coefficient and high fluorescence quantum yield. ${ }^{21}$ In the fluorescence spectra measured with a spectrofluorometer by using an emission scan mode, the emission peak of Cy5.5Lf-SPIO micelles was $689 \mathrm{~nm}$, which was the same as the pure Cy5.5 (Figure 4A). This suggested that the Cy5.5 was successfully conjugated to SPIO micelles and the Cy5.5Lf-SPIO micelles were suitable for fluorescence imaging. The fluorescence intensity of Cy5.5-Lf-SPIO micelles with various iron concentrations were measured with the IVIS Lumina XR system (Figure 4B). The results showed a positive linear correlation between the fluorescence intensity and the iron mass (Figure 4C).

\section{In vitro studies}

The ability of MRI and fluorescence imaging of Cy5.5-LfSPIO micelles at the cellular level was detected in rat C6 glioma cells. Along with the increasing iron concentration, a gradual MR-signal decay was observed (Figure 5A). 
A
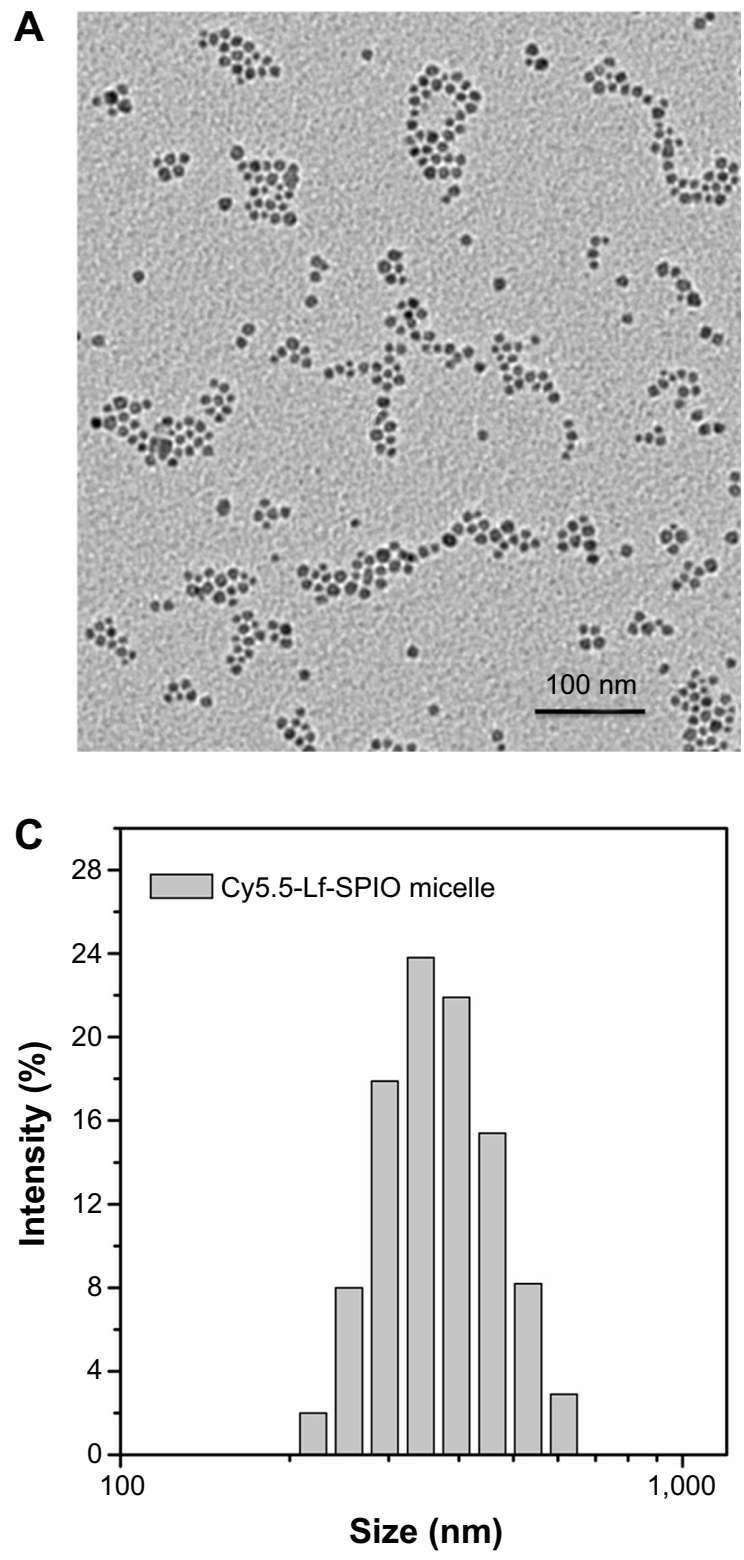

B
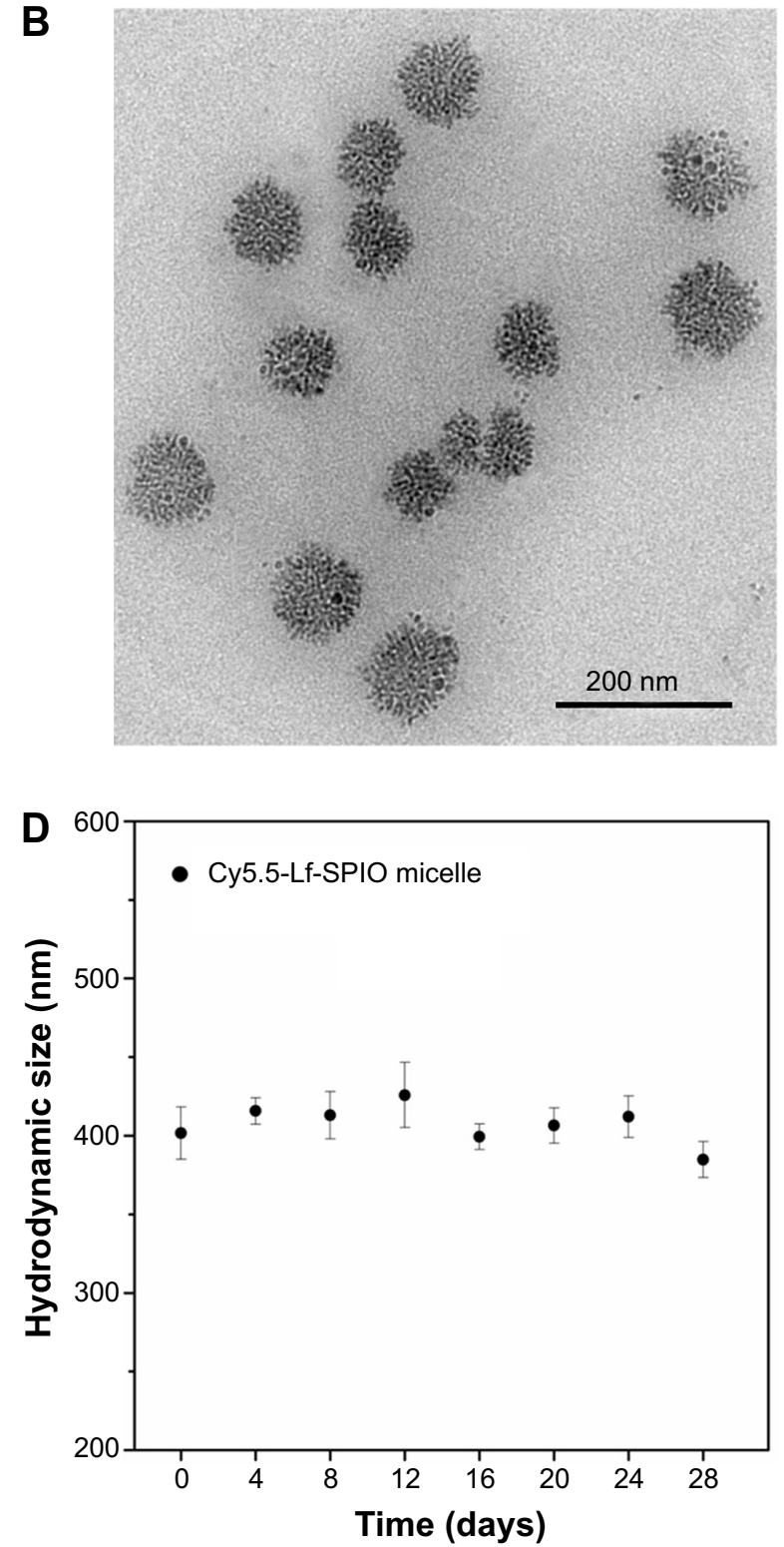

Figure 2 Morphology and size distribution of the materials.

Notes: (A) TEM image of SPIO. (B) TEM image of Cy5.5-Lf-SPIO micelles. (C) Hydrodynamic size distribution of Cy5.5-Lf-SPIO micelles at $25^{\circ} \mathrm{C}$. (D) Long-term stability based on hydrodynamic size change in phosphate-buffered saline over time at room temperature.

Abbreviations: TEM, transmission electron microscopy; Lf, lactoferrin; SPIO, superparamagnetic iron oxide.

Compared with the signal decay of the Cy5.5-SPIO micelle group, the signal decay of the Cy5.5-Lf-SPIO micelle group was much greater at the same iron concentration. This suggested that Cy5.5-Lf-SPIO micelles could be used as a contrast agent in vitro, and Lf targeted the C6 cells to improve the cellular uptake on the micelles in glioma cells.

From the fluorescence images obtained by the IVIS Lumina XR system (Figure 5B), with increasing iron concentration, there was a gradual enhancement of the fluorescence signal in the Cy5.5-Lf-SPIO micelle group. Compared to the Cy5.5-Lf-SPIO micelle group, there was no significant change in the Cy5.5-SPIO micelle group with increasing iron concentration, which was similar to the results obtained by MRI. To make a further comparison between the two groups, confocal microscopy was used to confirm the targeting ability of the nanoparticles (Figure 5C). In the Cy5.5-LfSPIO micelle group, there was a strong fluorescence signal throughout the cytoplasm in the $\mathrm{C} 6$ cells. However, a weak fluorescence signal was observed in the $\mathrm{C} 6$ cells treated with Cy5.5-SPIO micelles. All these results strongly suggested that Cy5.5-Lf-SPIO micelles could target rat C6 glioma cells and function well as a fluorescent probe. 
A

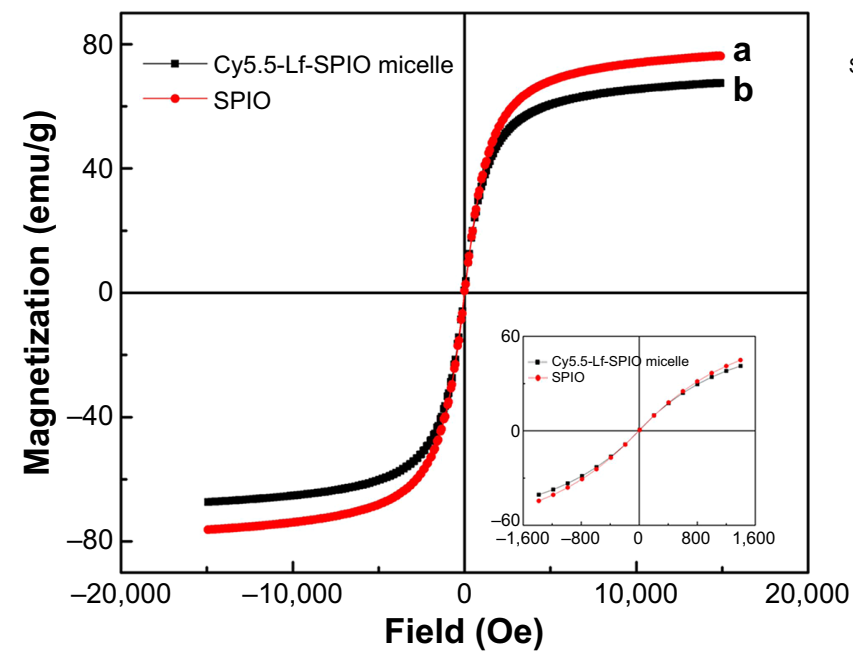

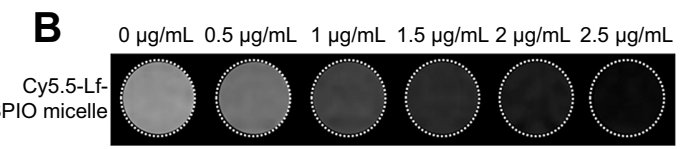

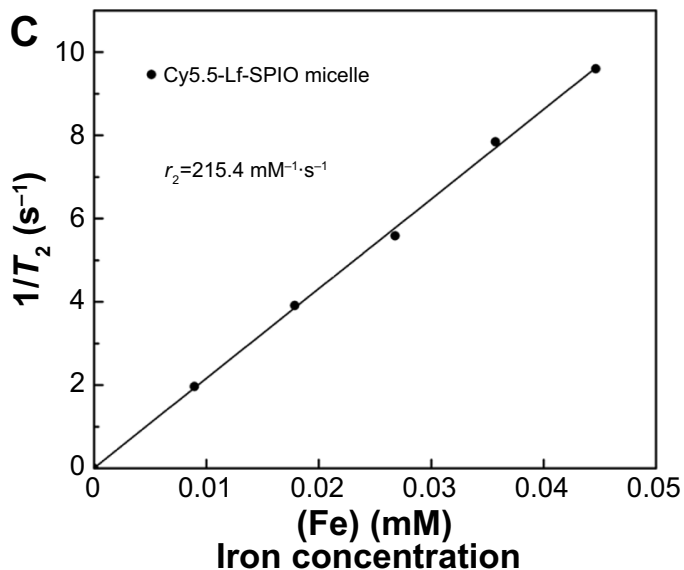

Figure 3 Magnetic properties and MR imaging abilities of the materials.

Notes: (A) Magnetization curves at $300 \mathrm{~K}$. (B) $T_{2}$-weighted MR images of the Cy5.5-Lf-SPIO micelles. (C) Relaxivities $\left(r_{2}\right)$ of the Cy5.5-Lf-SPIO micelles measured at 300 K. Abbreviations: MR, magnetic resonance; Lf, lactoferrin; SPIO, superparamagnetic iron oxide.

\section{In vivo studies}

The MR images in Figure 6A were obtained by a $3.0 \mathrm{~T}$ human MRI scanner. The rats bearing $\mathrm{C} 6$ homografts were used to evaluate the ability of the nanoparticles applied as a contrast agent. After injection with Cy5.5-Lf-SPIO micelles at a dosage of $12 \mathrm{mg} \mathrm{Fe} / \mathrm{kg}$, a significant contrast enhancement (darkening) was observed at 24 hours postinjection, and the range of high signal intensity expanded at 48 hours postinjection (glioma site indicated by the red circle). However, in the Cy5.5-SPIO micelle group, there was no apparent change exhibited on MR images of the rat brain at a dosage of $12 \mathrm{mg} \mathrm{Fe} / \mathrm{kg}$. The relative signal enhancement of the region of interest in the $T_{2}$-weighted image was evaluated through comparison between the tumor regions and the healthy muscle regions of the rats (Figure 6B). For the positive group, the relative signal enhancement of the tumor site at 48 hours reached $30 \%-67 \%$, while this was only $14 \%-26 \%$ in the negative group. For further verification of whether the relative signal enhancement was caused by the nanoparticles or not, the brain-tumor slices were stained

\section{A}

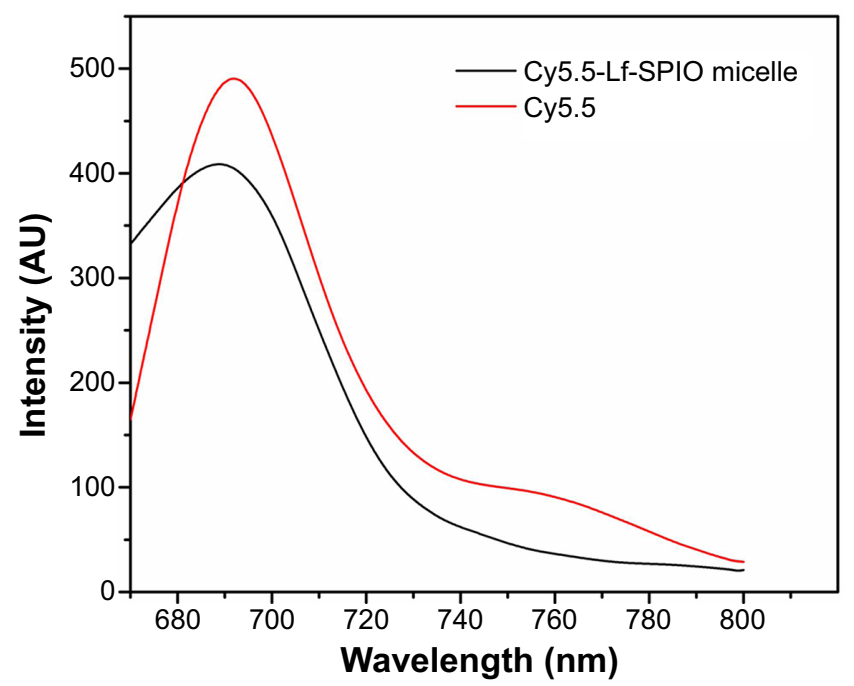

B

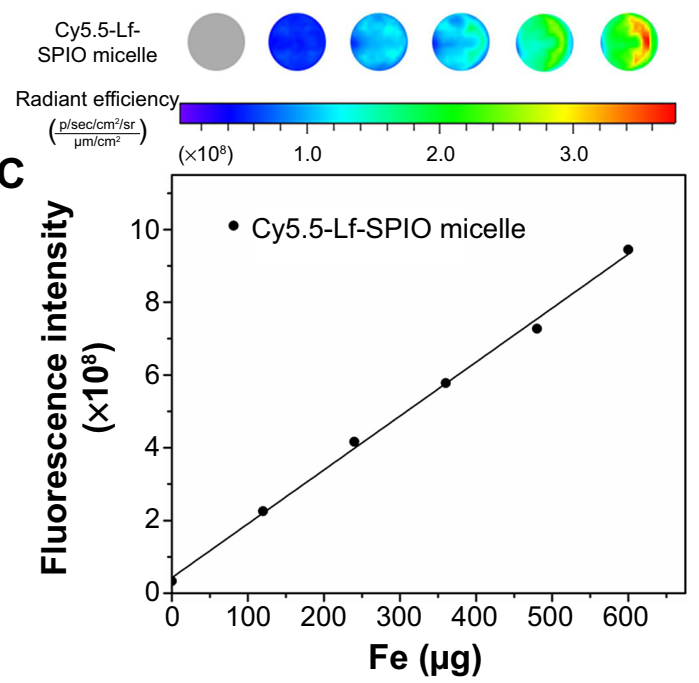

Figure 4 Fluorescence imaging abilities of the materials.

Notes: (A) Fluorescence spectra at the excitation wavelength of $640 \mathrm{~nm}$ at room temperature. (B) Fluorescent image of the Cy5.5-Lf-SPIO micelles. (C) The correlation between fluorescence intensity and the mass of the iron.

Abbreviations: Lf, lactoferrin; SPIO, superparamagnetic iron oxide. 
A $0 \mu \mathrm{g} / \mathrm{mL} \quad 5 \mu \mathrm{g} / \mathrm{mL} \quad 10 \mu \mathrm{g} / \mathrm{mL} \quad 15 \mu \mathrm{g} / \mathrm{mL} \quad 20 \mu \mathrm{g} / \mathrm{mL} \quad 25 \mu \mathrm{g} / \mathrm{mL}$

\section{Cy5.5-Lf- SPIO micelle \\ Cy5.5-SPIO micelle}

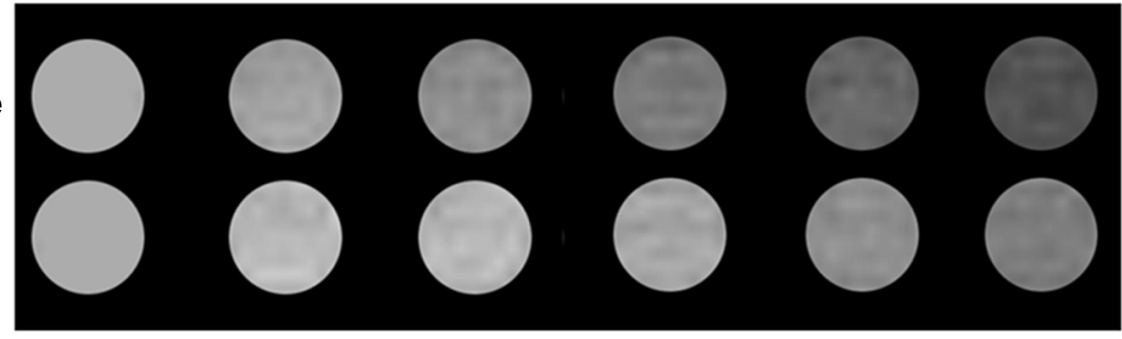

B $0 \mu \mathrm{g} / \mathrm{mL} \quad 5 \mu \mathrm{g} / \mathrm{mL} \quad 10 \mu \mathrm{g} / \mathrm{mL} \quad 15 \mu \mathrm{g} / \mathrm{mL} \quad 20 \mu \mathrm{g} / \mathrm{mL} \quad 25 \mu \mathrm{g} / \mathrm{mL}$

\section{Cy5.5-Lf- SPIO micelle}

\section{Cy5.5-SPIO micelle}

Radiant efficiency $\left(\frac{\mathrm{p} / \mathrm{sec} / \mathrm{cm}^{2} / \mathrm{sr}}{\mu \mathrm{m} / \mathrm{cm}^{2}}\right)$
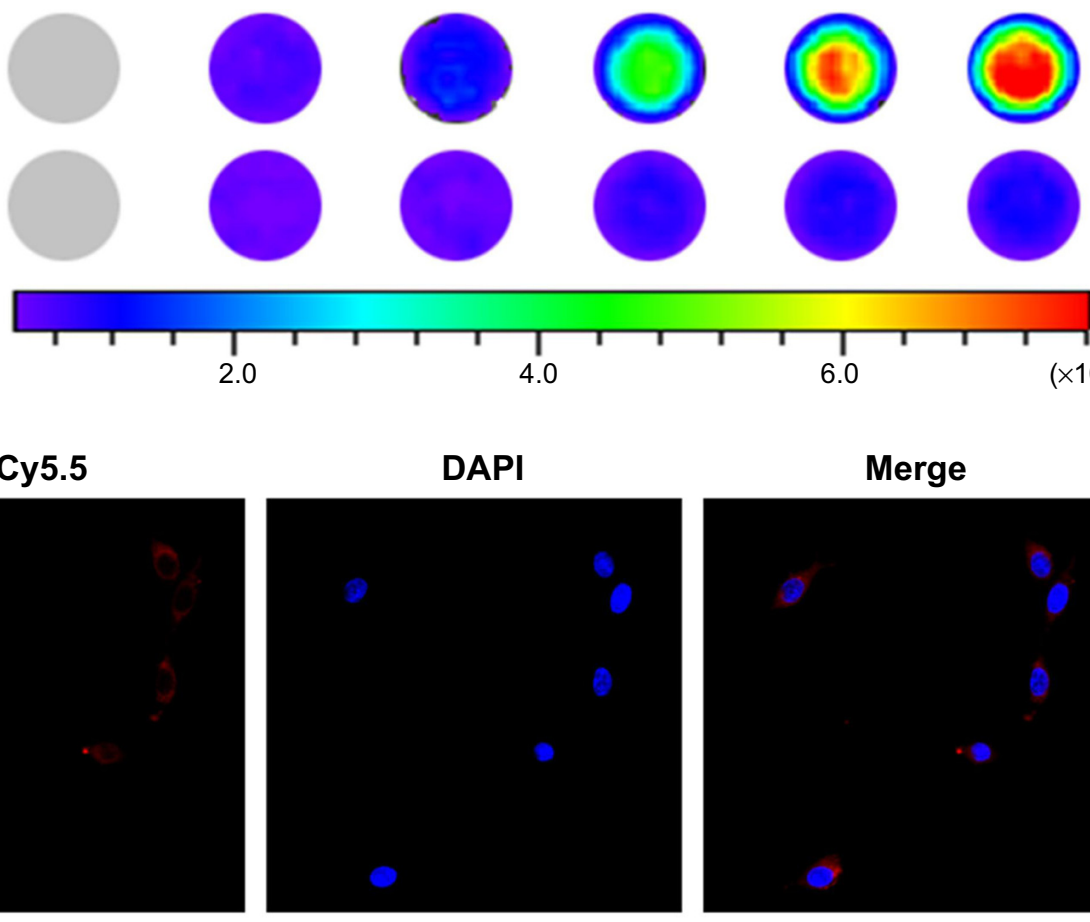

C
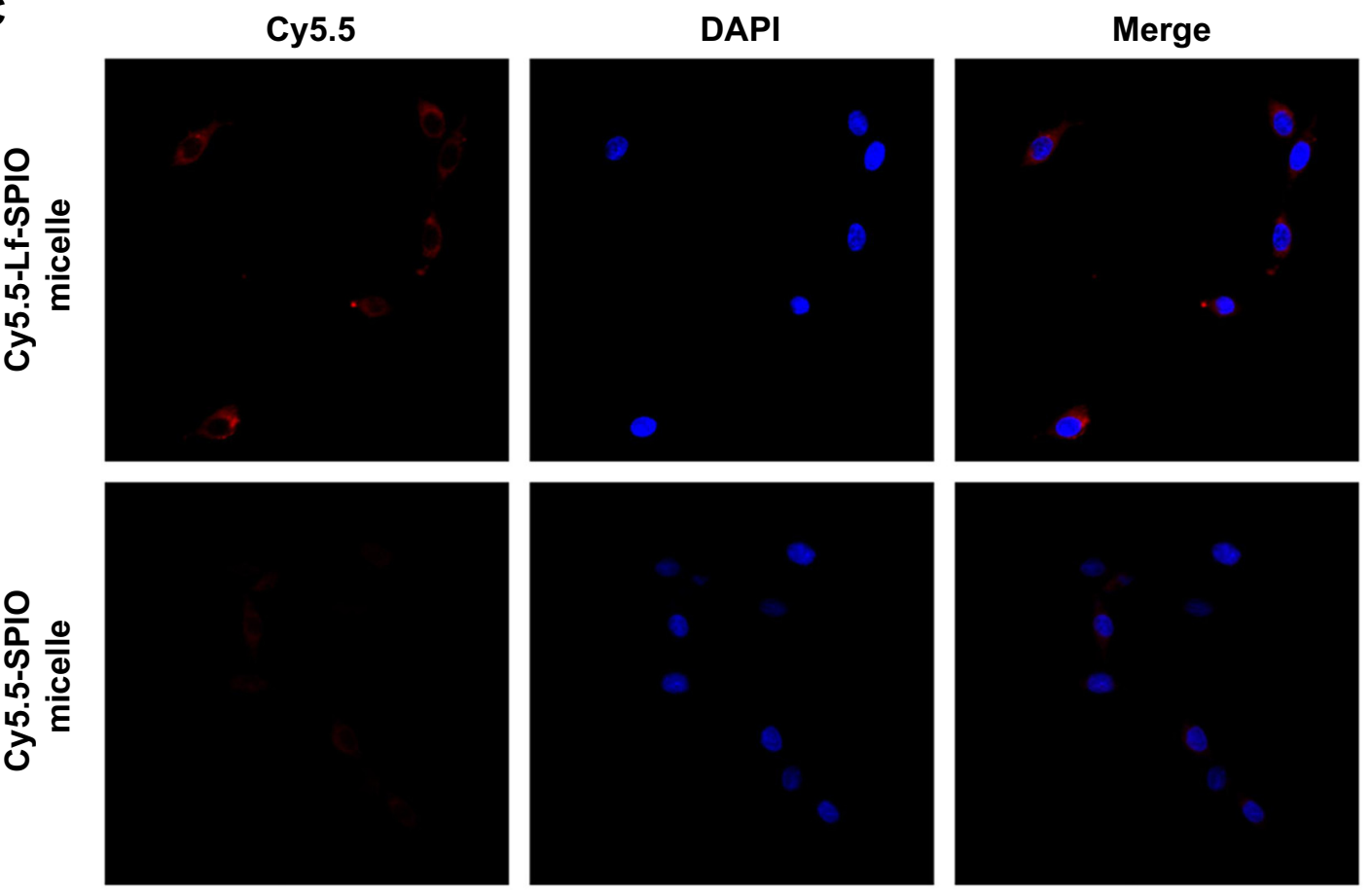

Figure $\mathbf{5}$ In vitro study of the materials.

Notes: (A) In vitro $T_{2}$-weighted magnetic resonance images of $\mathrm{C} 6$ cells with different treatments. (B) Fluorescence images of $C 6$ cells with different treatments. (C) Confocal fluorescence images of $C 6$ cells with different treatments (400X).

Abbreviations: Lf, lactoferrin; SPIO, superparamagnetic iron oxide; DAPI, 4',6-diamidino-2-phenylindole.

by Prussian blue to indicate the location of the iron. As shown in Figure 6C, in the Cy5.5-Lf-SPIO micelle group, the iron (blue dots) accumulated in the tumor tissues. Nevertheless, little iron was observed in the samples treated with Cy5.5-SPIO micelles. All these results suggested that Cy5.5-Lf-SPIO micelles targeted the brain tumor and functioned well as an MRI contrast agent for preoperative MRI diagnosis of the glioma.

To test the capability of Cy5.5-Lf-SPIO micelles utilized as an optical contrast agent (Figure 7A), ex vivo NIR fluorescence 
A
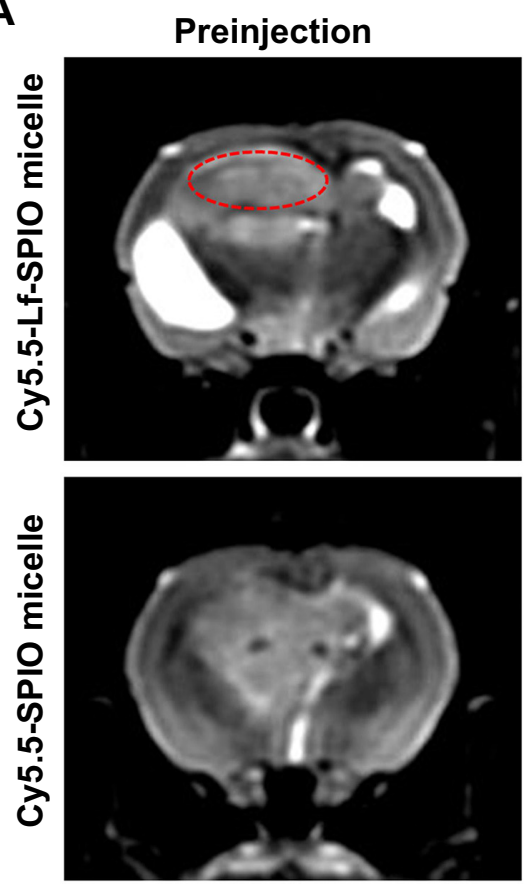

B

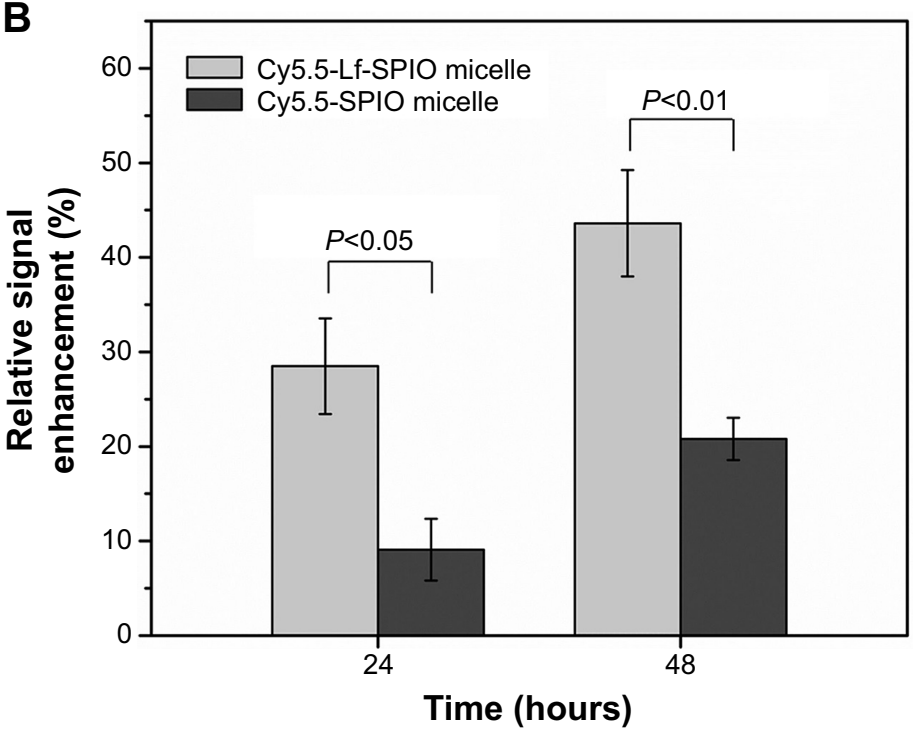

After 24 hours
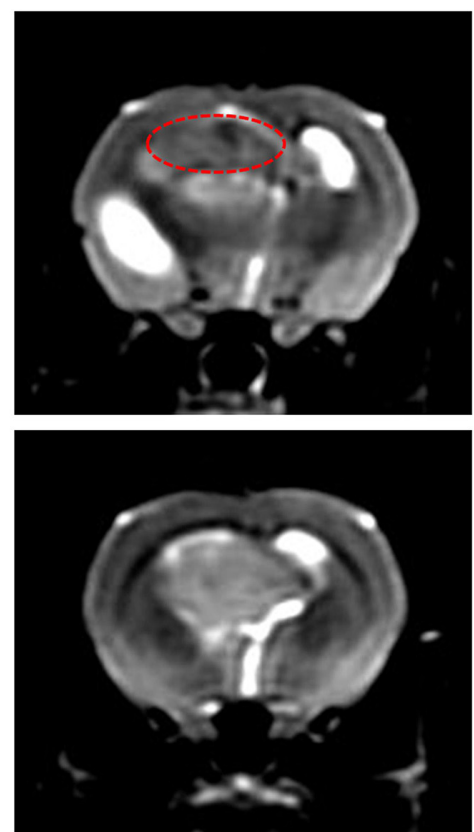

C
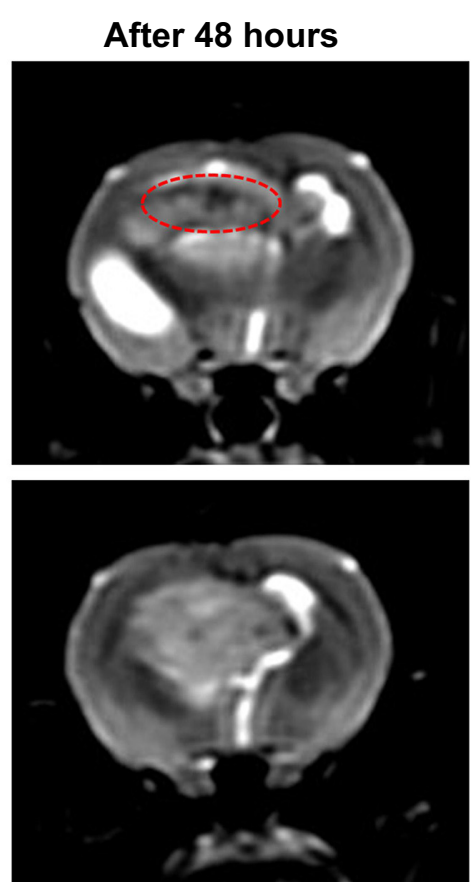

Prussian blue staining

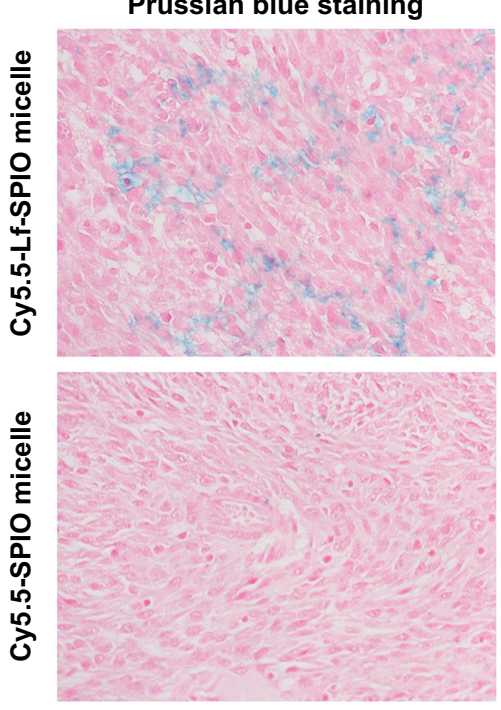

Figure 6 In vivo study on magnetic resonance imaging.

Notes: (A) In vivo $T_{2}$-weighted magnetic resonance images of rat brain bearing C6 glioma ( $\mathrm{n}=6$ ). Upper row, acquired after administration of Cy5.5-Lf-SPIO micelles ( 2 mg $\mathrm{Fe} / \mathrm{kg}$ ); lower row, acquired after administration of Cy5.5-SPIO micelles ( $12 \mathrm{mg} \mathrm{Fe} / \mathrm{kg}$ ). (B) The relative signal enhancement of the brain tumor in the $T_{2}$-weighted image at 24 and 48 hours (expressed as means \pm standard error of mean $[n=6])$. (C) Histological sections of glioma with Prussian blue staining (400x).

Abbreviations: Lf, lactoferrin; SPIO, superparamagnetic iron oxide.

images of the rats' brains were analyzed by the IVIS Lumina XR system. As shown in Figure 7A, there was a distinct fluorescence signal in the middle of the brain obtained from the rats treated with Cy5.5-Lf-SPIO micelles. Abnormal cell density and morphology of tumor tissue were observed in the H\&E-stained image (the purple part in the brain slice). H\&E staining verified the location and the boundary of the brain tumor, which corresponded with the results from the NIR fluorescence images. However, for the negative group, there was no detectable fluorescence signal at the tumor site. The average fluorescence intensity of the brain-tumor tissue and the normal brain tissue was measured (Figure 7B). In the positive group, the average fluorescence intensity of the brain tumor was 3.8 times higher than that of normal brain tissue, which indicated that the NIR fluorescence was sensitive enough to discriminate the tumor tissue from the normal brain tissue. 
A

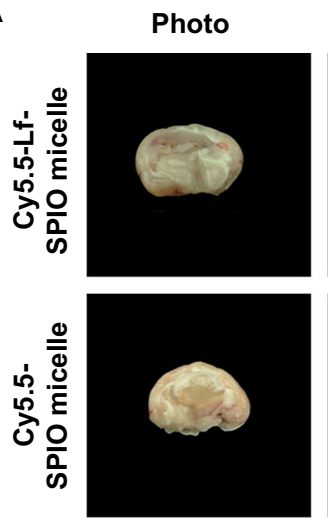

Cy5.5
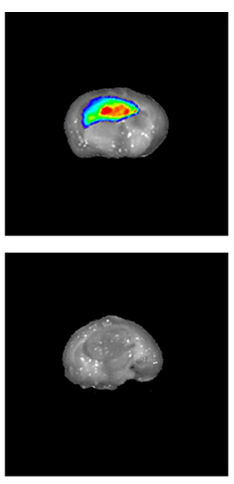

H\&E staining
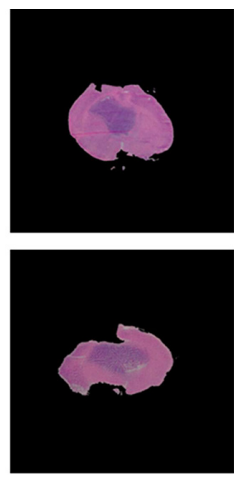

B

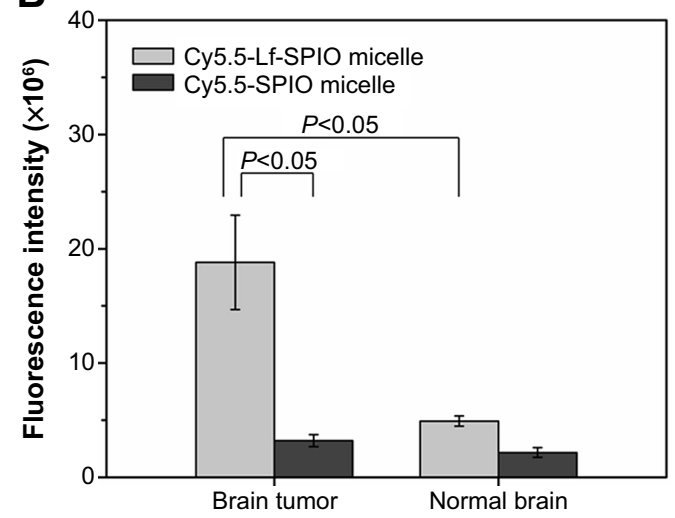

Figure 7 Ex vivo study of the brain tissue.

Notes: (A) Ex vivo fluorescence images and H\&E-staining images of rat brain bearing $\mathrm{C} 6$ glioma at 48 hours postinjection. (B) The average fluorescence intensity of the brain tumor and the normal brain. Results expressed as means \pm standard error of mean $(n=6)$.

Abbreviations: Lf, lactoferrin; SPIO, superparamagnetic iron oxide; H\&E, hematoxylin and eosin.

The location of the nanoparticles was investigated by confocal fluorescence microscopy. The cell nucleus was stained with DAPI. From the confocal fluorescence images (Figure 8 ), in the brain slices of the rats treated with Cy5.5-Lf-SPIO micelles, apparent red fluorescence was observed at high-celldensity sites, which proved to be the tumor site. This suggested that the nanoparticles were retained at the tumor site and could distinctly outline the margin of the glioma on the optical image. However, no distinct Cy5.5 fluorescent signal was detected in the negative group. All these results were consistent with those obtained from the MR images, which suggested that the nanoparticles could selectively target the glioma. Due to the accumulation of the nanoparticles, the location of the brain tumor was defined clearly on the optical image. Based on these results, it was supposed that Cy5.5-Lf-SPIO micelles could be applied for guiding the resection of the glioma in intraoperative fluorescence imaging.

The rats' organs were collected for biodistribution study. The IVIS Lumina XR system was used to collect the optical images of the rat organs. The result is shown in the Figure S2. The fluorescence signal was mainly distributed in the brain-tumor site. There were also some weaker signals in the liver and kidney which are usually known as the organs related to the metabolism of nanoparticle.

\section{Biocompatibility studies}

Cytotoxicity was evaluated by MTT assays in vitro (Figure 9). The results suggested that the metabolic activity of the
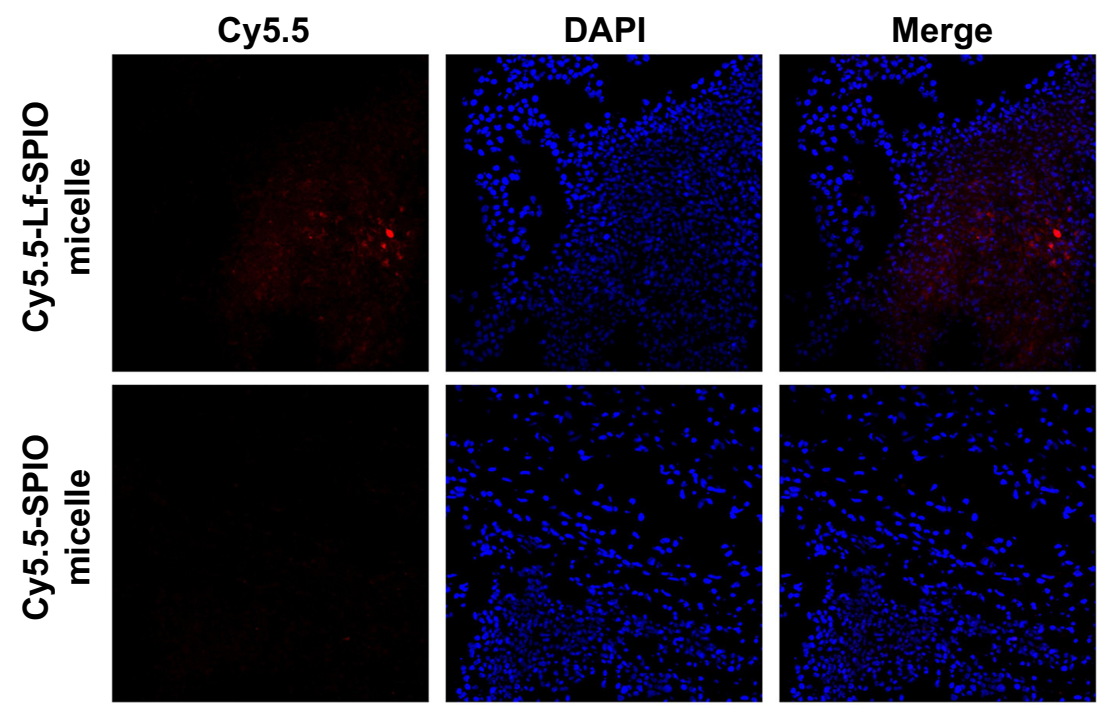

Figure 8 The confocal fluorescence images of the brain slices.

Notes: Upper row, sections of the group with treatment of Cy5.5-Lf-SPIO micelles (I00X); lower row, sections of the group with treatment of Cy5.5-SPIO micelles (I00X). Abbreviations: Lf, lactoferrin; SPIO, superparamagnetic iron oxide; DAPI, 4',6-diamidino-2-phenylindole. 


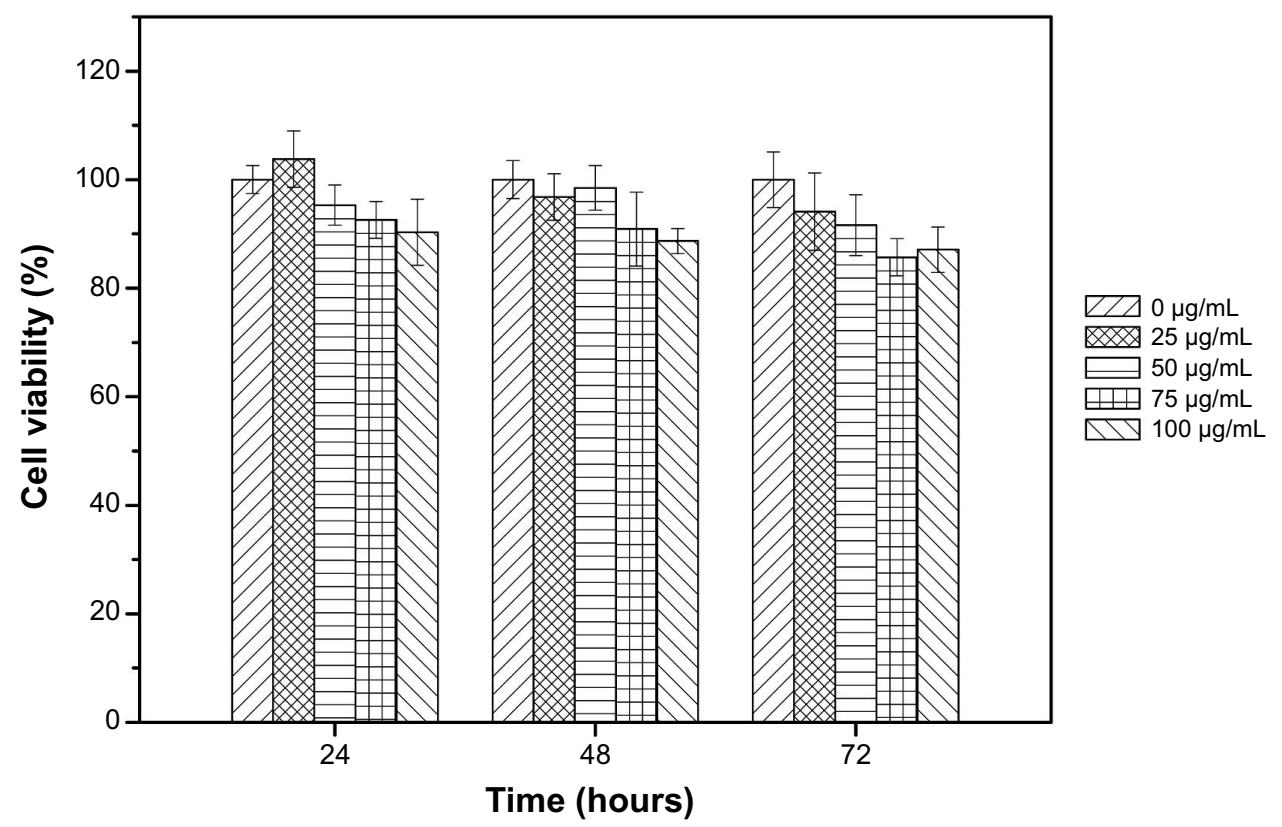

Figure 9 Viabilities of NIH-3T3 cells treated with Cy5.5-Lf-SPIO micelles in cytotoxicity studies. Results expressed as means \pm standard error of mean. Abbreviations: Lf, lactoferrin; SPIO, superparamagnetic iron oxide.

NIH-3T3 cells was not affected by the Cy5.5-Lf-SPIO micelles when the micelles were added in the range of $0-100$ $\mu \mathrm{g} / \mathrm{mL}$ (iron concentration).

\section{Discussion}

Gliomas are the most common and deadly brain tumor. Surgical resection is the primary treatment mode for glioma. The resection region is closely related to the survival rate and life quality. However, due to the destructiveness, infiltration, and invasiveness of gliomas, it is difficult to acquire a gross total resection of the brain tumor. Therefore, demarcation of the tumor margin is crucial for patients bearing gliomas. MRI is currently considered as the gold standard for glioma diagnosis. Nevertheless, it is not sensitive enough to delineate the tumor boundary clearly. Meanwhile, optical imaging, especially NIRF imaging, offers high sensitivity, and has entered the surgical theater to fill the gap between preoperative imaging and intraoperative reality. ${ }^{25}$ However, the application of optical imaging in the clinical field is limited, due to low spatial resolution. ${ }^{26}$ Therefore, the combination of MRI and optical imaging will cover the shortage of each single imaging mode and provide guidance for physicians at both the presurgical planning stage and the surgical resection stage.

As a consequence, a large variety of different approaches for the synthesis of nanoparticles exhibiting magnetic and fluorescent properties has been developed. Quantum dots are considered as an excellent fluorescent dye, because of their stable optical properties and controllable fluorescence spectrum. Gu et al coated FePt cores with CdS shells to generate heterodimers of nanoparticles, which exhibited both optical and magnetic properties. ${ }^{27}$ However, the toxicity of the quantum dots limits their biological application. ${ }^{28}$ Another approach is to couple the organic fluorophores to the iron oxide nanoparticles. Kircher et al synthesized iron oxide nanoparticles with the NIRF dye Cy5.5, which was attached to the dextran-coating layer of the nanoparticles. ${ }^{29}$ Nevertheless, due to the short interval between the emitting species and the magnetic one, fluorescence of the fluorophore would be strongly reduced or even quenched. ${ }^{15,24,30}$ To solve this problem, Cha et al designed an "activatable" multimodal imaging probe. In their study, the NIR fluorescent dye Cy5.5 was connected to the SPIO through a matrix metalloproteinase substrate. ${ }^{24}$ In a normal physiological environment, the Cy5.5 was quenched by the SPIO. At the tumor site, the substrate was hydrolyzed by matrix metalloproteinase 2. Cy5.5 was released from the SPIO, and was able to be used as an optical probe.

In this study (Figure 10), we developed novel optical/MRI bifunctional micelles for specific targeting of gliomas. The nanoparticles selectively accumulated in the brain tumors due to the tumor-targeting ligand $\mathrm{Lf}^{22,31,32}$ Encapsulation of the SPIO in the micelles can make the micelles be applied as an efficient MRI contrast agent. Meanwhile, with connection of the NIR fluorescence dye Cy5.5, the nanoparticles outlined the margin of the brain tumor through NIR fluorescence imaging with high sensitivity, which compensated for the inherent shortage, low sensitivity, of MRI. 


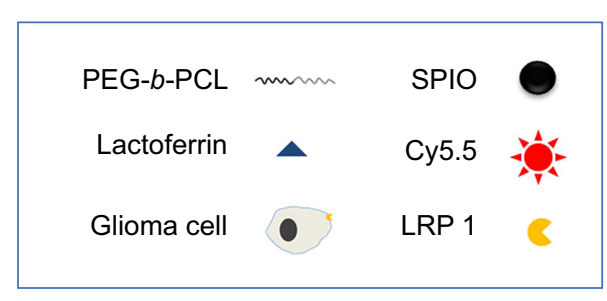

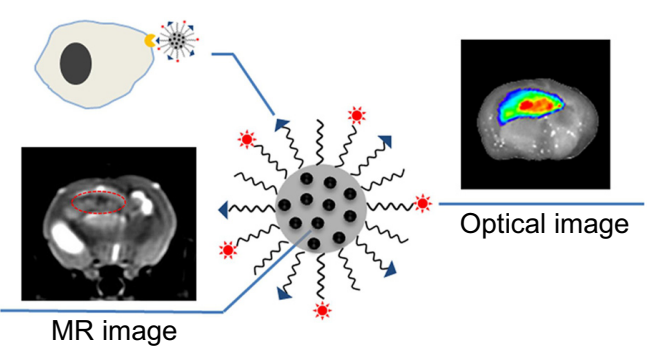

Figure 10 Illustration of the multiple functions of Cy5.5-Lf-SPIO micelles.

Abbreviations: Lf, lactoferrin; SPIO, superparamagnetic iron oxide; PEG-b-PCL, polyethylene glycol-block-polycaprolactone; MR, magnetic resonance.

As shown in the in vivo results, the tumor tissue was discriminated from the normal brain tissue in the NIR fluorescence image, and the location of the tumor corresponded highly with that indicated by the MR image and the H\&Estained image. In order to keep the optical property of the nanoparticles, control of spacing between the emitting species and the magnetic one is the key point. ${ }^{30}$ The polymer PEG- $b$-PCL with high molecular weight provided a long interval between the fluorescence dye and the SPIO, which prevented the quenching effect. With encapsulation by PEG- $b$-PCL, the hydrophobic SPIO formed a cluster that contributed to the increase of $T_{2}$ relaxivity. In Ai et al's study, single SPIO-containing micelles and the micelles with a cluster of SPIO particles encapsulated inside the hydrophobic core were compared. ${ }^{11}$ They found that the $T_{2}$ relaxivity of the latter reached $169 \mathrm{mM}^{-1} \cdot \mathrm{s}^{-1}$, while it was only $25.1 \mathrm{mM}^{-1} \cdot \mathrm{s}^{-1}$ for the single SPIO-containing micelles. They drew the conclusion that the $T_{2}$ relaxivity was directly proportional to the SPIO-loading density of the micelles and increased dramatically with SPIO clustering. In this study, we chose $\mathrm{PEG}_{5000}-b-\mathrm{PCL}_{15000}$ to encapsulate the SPIO. The long PCL segments would form a hydrophobic core, which could have higher affinity for SPIO and stabilize micelle formation with a very low critical micelle concentration. ${ }^{33}$ The micelles had a larger $T_{2}$ relaxivity $\left(215.4 \mathrm{mM}^{-1} \cdot \mathrm{s}^{-1}\right)$ than traditional SPIO-dextran contrast agents, such as Feridex $\left(120 \mathrm{mM}^{-1} \cdot \mathrm{s}^{-1}\right) .^{34}$ The high relaxivity of the nanoprobe will benefit the generation of detectable MR signals even when the nanoprobe concentration is low in brain tissues.

In addition, the PEG segment would increase the serum half-life of the nanoprobe in vivo. ${ }^{10-12,35}$ Veiseh et al reported that SPIO modified by PEG was detected by MRI even at 120 hours postinjection. ${ }^{10}$ In our research, compared to the results at 24 hours, the region of the hypointensity in the brain expanded at 48 hours postinjection, which indicated that the nanoparticles achieved a long blood half-life. Compared to SPIO, the $\mathrm{Gd}^{3+}$-based contrast agent, mentioned earlier as a common contrast agent for glioma, had the limitation of providing time-dependent images of tumor margins, since approximately $96 \%$ of $\mathrm{Gd}^{3+}$ would be excreted within 24 hours by renal glomerular filtration. ${ }^{7}$ Therefore, the long duration of the hypointensity at the tumor site with the micelles will be particularly valuable in assessing the effectiveness of the therapies after surgery or radiotherapy.

\section{Conclusion}

Overall, we have designed a multifunctional micelle which could be applied as an MRI contrast agent before operation and demarcate the boundary of the brain tumor on the fluorescence image during surgery with high sensitivity. The encapsulation of amphiphilic copolymer protected micelles' optical properties from obstruction by SPIO and made a contribution to the increase of the $T_{2}$ relaxivity with SPIO clustering. Owing to the ligand Lf, the nanoparticles specifically targeted brain tumor cells both in vitro and in vivo. The long duration of the hypointensity at the tumor site on the MR image is desirable for the diagnosis of glioma after surgery to assess the effectiveness of therapies.

\section{Acknowledgments}

This work was financially supported by a grant from National Basic Research Program of China (program 973, 2012CB932500), the National Science Foundation of China (NSFC, 31170960/C1007), and the National Program of Ministry of Science and Technology of China under the 12th Five-Year Plan (2011BAI08B10). We give thanks to the Analysis and Test Center of Huazhong University of Science and Technology. We also give special thanks to Professor Yuzeng Zhang (MOE Key Lab of Environment and Health, School of Public Health, Tongji Medical College, Huazhong University of Science and Technology) for help in the AAS analysis.

\section{Disclosure}

The authors report no conflicts of interest in this work. 


\section{References}

1. Tanaka S, Louis DN, Curry WT, Batchelor TT, Dietrich J. Diagnostic and therapeutic avenues for glioblastoma: no longer a dead end? Nat Rev Clin Oncol. 2013;10(1):14-26.

2. Koo YE, Reddy GR, Bhojani M, et al. Brain cancer diagnosis and therapy with nanoplatforms. Adv Drug Deliv Rev. 2006;58(14):1556-1577.

3. Sanai N, Berger MS. Glioma extent of resection and its impact on patient outcome. Neurosurgery. 2008;62(4):753-766.

4. Claes A, Idema AJ, Wesseling P. Diffuse glioma growth: a guerilla war. Acta Neuropathol. 2007;114(5):443-458.

5. Zhou J, Tryggestad E, Wen Z, et al. Differentiation between glioma and radiation necrosis using molecular magnetic resonance imaging of endogenous proteins and peptides. Nat Med. 2011;17(1):130-134.

6. Giesel FL, Mehndiratta A, Essig M. High-relaxivity contrast-enhanced magnetic resonance neuroimaging: a review. Eur Radiol. 2010;20(10): 2461-2474.

7. Crich $\mathrm{G}$, Cabella $\mathrm{C}$, Barge A, et al. In vitro and in vivo magnetic resonance detection of tumor cells by targeting glutamine transporters with Gd-based probes. J Med Chem. 2006;49(16):4926-4936.

8. Laurent S, Forge D, Port M, et al. Magnetic iron oxide nanoparticles: synthesis, stabilization, vectorization, physicochemical characterizations, and biological applications. Chem Rev. 2008;108(6):2064-2110.

9. Xu Z, Shen C, Hou Y, Gao H, Sun S. Oleylamine as both reducing agent and stabilizer in a facile synthesis of magnetite nanoparticles. Chem Mater. 2009;21(9):1778-1780.

10. Veiseh O, Sun C, Fang C, et al. Specific targeting of brain tumors with an optical/magnetic resonance imaging nanoprobe across the bloodbrain barrier. Cancer Res. 2009;69(15):6200-6207.

11. Ai H, Flask C, Weinberg B, et al. Magnetite-loaded polymeric micelles as ultrasensitive magnetic-resonance probes. Adv Mater. 2005;17(16): 1949-1952.

12. Weissleder R, Bogdanov A, Neuwelt EA, Papisov M. Long-circulating iron oxides for MR imaging. Adv Drug Deliv Rev. 1995;16(2):321-334.

13. Cheng D, Hong G, Wang W, et al. Nonclustered magnetite nanoparticle encapsulated biodegradable polymeric micelles with enhanced properties for in vivo tumor imaging. J Mater Chem. 2011;21(13):4796-4804.

14. Kubben PL, ter Meulen KJ, Schijns OE, ter Laak-Poort MP, van Overbeeke JJ, van Santbrink H. Intraoperative MRI-guided resection of glioblastoma multiforme: a systematic review.Lancet Oncol. 2011;12(11): 1062-1070.

15. Louie A. Multimodality imaging probes: design and challenges. Chem Rev. 2010;110(5):3146-3195.

16. Yan H, Wang L, Wang J, et al. Two-order targeted brain tumor imaging by using an optical/paramagnetic nanoprobe across the blood-brain barrier. ACS Nano. 2011;6(1):410-420.

17. Tsugu A, Ishizaka H, Mizokami Y, et al. Impact of the combination of 5-aminolevulinic acid-induced fluorescence with intraoperative magnetic resonance imaging-guided surgery for glioma. World Neurosurg. 2011;76(1-2):120-127.

18. Tonn JC, Stummer W. Fluorescence-guided resection of malignant gliomas using 5-aminolevulinic acid: practical use, risks, and pitfalls. Clin Neurosurg. 2007;55:20-26.
19. Floeth FW, Sabel M, Ewelt C, et al. Comparison of 18F-FET PET and 5-ALA fluorescence in cerebral gliomas. Eur J Nucl Med Mol Imaging. 2011;38(4):731-741.

20. Weissleder R, Ntziachristos V. Shedding light onto live molecular targets. Nat Med. 2003;9(1):123-128.

21. Luo S, Zhang E, Su Y, Cheng T, Shi C. A review of NIR dyes in cancer targeting and imaging. Biomaterials. 2011;32(29):7127-7138.

22. Xie H, Zhu Y, Jiang W, et al. Lactoferrin-conjugated superparamagnetic iron oxide nanoparticles as a specific MRI contrast agent for detection of brain glioma in vivo. Biomaterials. 2011;32(2):495-502.

23. Jiang LY, Zhou Q, Mu KT, et al. pH/temperature sensitive magnetic nanogels conjugated with Cy5.5-labled lactoferrin for MR and fluorescence imaging of glioma in rats. Biomaterials. 2013;34(30): 7418-7428.

24. Cha EJ, Jang ES, Sun IC, et al. Development of MRI/NIRF 'activatable' multimodal imaging probe based on iron oxide nanoparticles. $J$ Control Release. 2011;155(2):152-158.

25. Vahrmeijer AL, Hutteman M, van der Vorst JR, van de Velde CJ, Frangioni JV. Image-guided cancer surgery using near-infrared fluorescence. Nat Rev Clin Oncol. 2013;10(9):507-518.

26. Cho HJ, Yoon HY, Koo H, et al. Hyaluronic acid-ceramide-based optical/MR dual imaging nanoprobe for cancer diagnosis. J Control Release. 2012;162(1):111-118.

27. Gu H, Zheng R, Zhang X, Xu B. Facile one-pot synthesis of bifunctional heterodimers of nanoparticles: a conjugate of quantum dot and magnetic nanoparticles. J Am Chem Soc. 2004;126(18):5664-5665.

28. Howes P, Green M, Bowers A, et al. Magnetic conjugated polymer nanoparticles as bimodal imaging agents. J Am Chem Soc. 2010;132(28): 9833-9842.

29. Kircher MF, Mahmood U, King RS, Weissleder R, Josephson L. A multimodal nanoparticle for preoperative magnetic resonance imaging and intraoperative optical brain tumor delineation. Cancer Res. 2003; 63(23):8122-8125.

30. Bigall NC, Parak WJ, Dorfs D. Fluorescent, magnetic and plasmonichybrid multifunctional colloidal nano objects. Nano Today. 2012;7(4): 282-296.

31. Demeule M, Currie JC, Bertrand Y, et al. Involvement of the lowdensity lipoprotein receptor-related protein in the transcytosis of the brain delivery vector angiopep-2. J Neurochem. 2008;106(4): 1534-1544.

32. Strickland DK, Gonias SL, Argraves WS. Diverse roles for the LDL receptor family. Trends Endocrinol Metab. 2002;13(2):66-74.

33. Savic R, Luo LB, Eisenberg A, Maysinger D. Cellular distribution of nonionic micelles - response. Science. 2004;303(5658):627-628.

34. Na HB, Song IC, Hyeon T. Inorganic nanoparticles for MRI contrast agents. Adv Mater. 2009;21(21):2133-2148.

35. Sun C, Du K, Fang C, et al. PEG-mediated synthesis of highly dispersive multifunctional superparamagnetic nanoparticles: their physicochemical properties and function in vivo. ACS Nano. 2010;4(4):2402-2410. 


\section{Supplementary materials FTIR spectrum}

The Fourier-transform infrared (FTIR) spectrum was used to achieve qualitative analysis of the lactoferrin ( $\mathrm{Lf}$ ) conjugation. As shown in the FTIR spectra (Figure S1), in the pure Lf group and Cy5.5-Lf-superparamagnetic iron oxide (SPIO) micelle group, the bands around 3,400 $\mathrm{cm}^{-1}$ were for the acylamino-stretching vibration, which indicated that the Lf existed. In the Cy5.5-Lf-SPIO micelle group, the bands around $700 \mathrm{~cm}^{-1}$ appeared due to $\mathrm{S}-\mathrm{C}$ bond formation, which indicated that Lf successfully conjugated to micelles.

\section{Biodistribution of the nanoparticles}

The animals treated with Cy5.5-Lf-SPIO micelles were killed at 48 hours postinjection. The IVIS Lumina XR system was used to collect optical images of the rats' organs. As shown in Figure S2, the fluorescence signals were mainly distributed in the brain-tumor site. There were also some weaker signals in the liver and kidney, which are usually known as the organs related to the metabolism of nanoparticle.

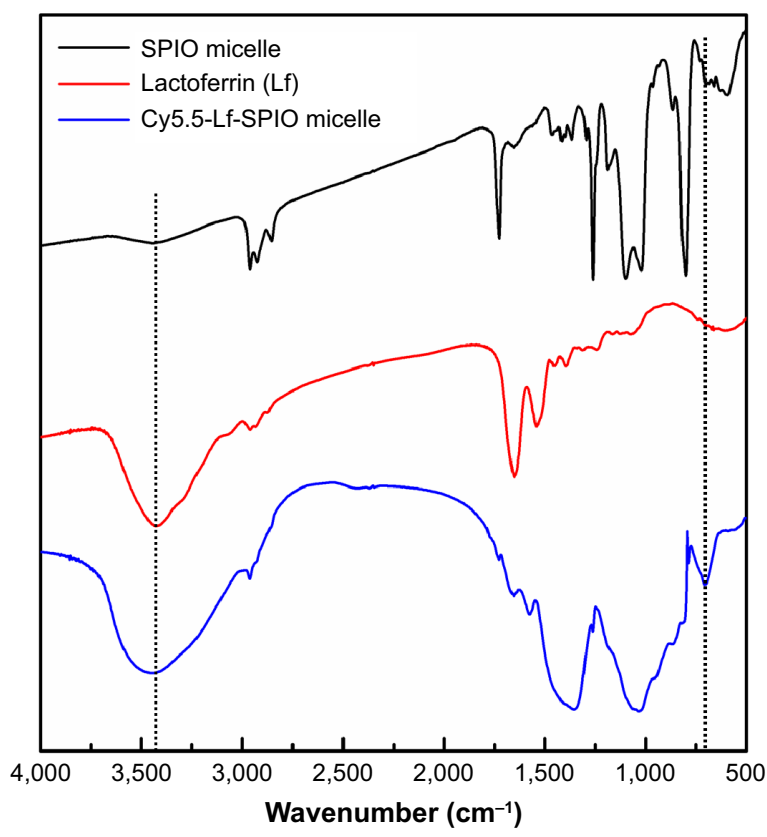

Figure SI Fourier-transform infrared spectra of SPIO micelles, lactoferrin, and Cy5.5-Lf-SPIO micelle.

Abbreviation: SPIO, superparamagnetic iron oxide.

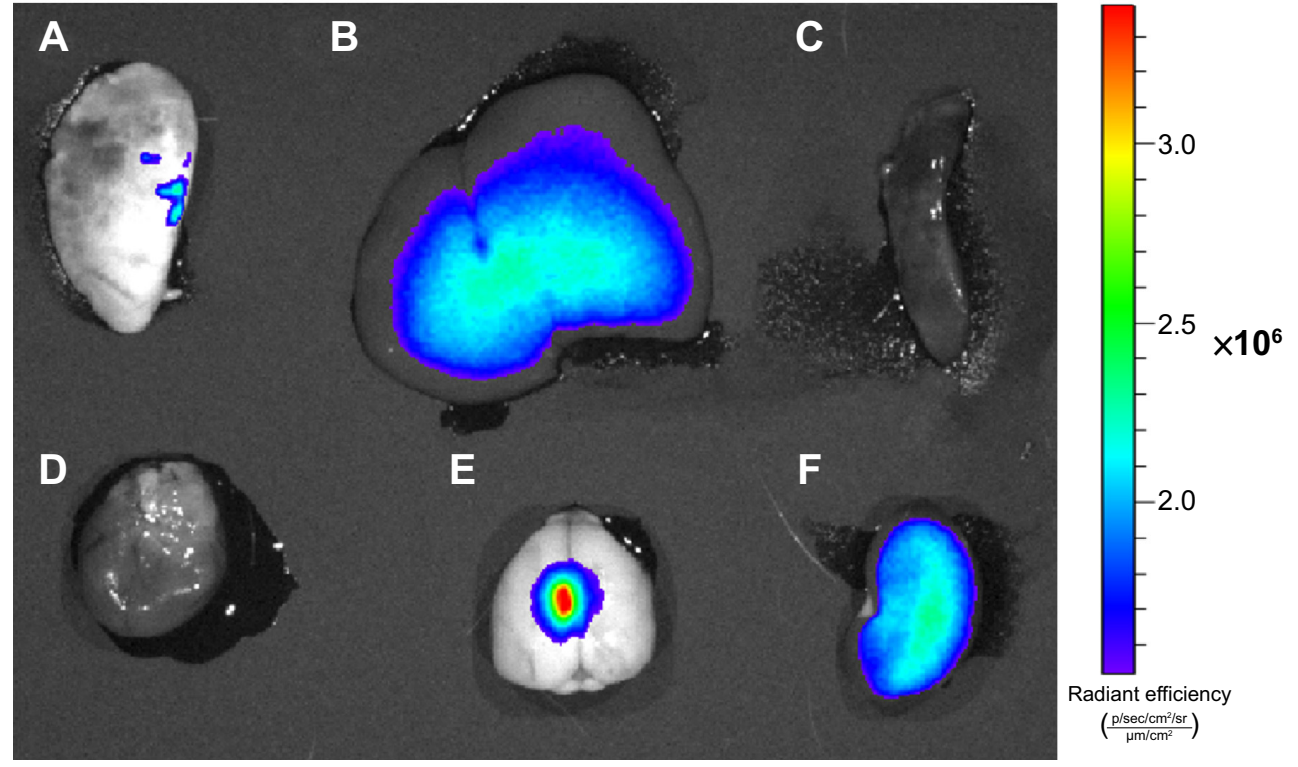

Figure S2 Optical images of rat organs.

Notes: (A) Lung; (B) liver; (C) spleen; (D) heart; (E) brain; (F) kidney.

\section{Publish your work in this journal}

The International Journal of Nanomedicine is an international, peerreviewed journal focusing on the application of nanotechnology in diagnostics, therapeutics, and drug delivery systems throughout the biomedical field. This journal is indexed on PubMed Central, MedLine, CAS, SciSearch $\AA$, Current Contents $\AA /$ Clinical Medicine,
Journal Citation Reports/Science Edition, EMBase, Scopus and the Elsevier Bibliographic databases. The manuscript management system is completely online and includes a very quick and fair peer-review system, which is all easy to use. Visit http://www.dovepress.com/ testimonials.php to read real quotes from published authors. 